\title{
Balamuthia mandrillaris infection
}

The concept that certain small free-living amoebae such as Acanthamoeba have the potential to cause disease in animals, including man, was suggested by Culbertson in 1961 [1]. This observation was based on remarkable experiments conducted in the late 1950s during the manufacture of polio vaccine at the Eli Lilly laboratories in Indianapolis, IN, USA $[1,2]$. A few years later Fowler and Carter in Australia [3] and Butt [4] in the USA described the first human cases of amoebic meningo-encephalitis caused by Naegleria fowleri. Since then several hundred cases of central nervous system (CNS) disease caused by Acanthamoeba spp., N. fowleri and Balamuthia mandrillaris have been reported worldwide [5-7]. Several cases in animals other than man have also been reported $[8,9]$. Infection is almost invariably fatal. The CNS disease produced by $N$. fowleri is called primary amoebic meningo-encephalitis and occurs principally in immunocompetent children and young adults with a history of contact with fresh water; infection is fulminating and nearly always fatal. The infection caused by Acanthamoeba spp. or B. mandrillaris is called granulomatous amoebic encephalitis. Acanthamoeba encephalitis occurs principally in immunosuppressed individuals, chronic alcoholics and debilitated persons; B. mandrillaris causes encephalitis in the very young or very old and in immunosuppressed individuals. Acanthamoeba spp. also cause a non-fatal, but vision-threatening keratitis of the human eye [5-7]. The true incidence of amoebic encephalitis is not exactly known. As of 1 Aug. 2000, >190 cases of meningo-encephalitis due to $N$. fowleri have been reported worldwide, 95 in the USA alone. More than 120 cases of granulomatous encephalitis due to Acanthamoeba spp. (84 in the USA, including at least 50 patients with HIV) and $>3000$ cases of Acanthamoeba keratitis have been reported. There have been $>85$ additional reports of encephalitis due to $B$. mandrillaris; 40 of these occurred in the USA, and include $>10$ patients with HIV. The actual number is probably higher, as the diagnosis is usually made at autopsy, which is seldom done in many countries [5].

Until 1989, the identification of the causative agent of granulomatous amoebic encephalitis, in the absence of culture isolation or immunohistological assay, was based on the presence of amoebic cysts in tissue sections, as $N$. fowleri does not produce cysts in tissue whereas Acanthamoeba does. Hence many cases were identified as due to Acanthamoeba solely on the basis of cysts in the tissue sections. Isolation of $B$. mandrillaris - initially from the brain tissue of a mandrill that died of encephalitis in the San Diego Wild Animal Park, and subsequently from human and other animal brain tissue - and the production of specific anti- $B$. mandrillaris serum, were instrumental in differentiating Balamuthia from Acanthamoeba encephalitis $[10,11]$.

\section{Protozoology, cultural characteristics, and epidemiology}

B. mandrillaris, like Acanthamoeba, has a vegetative trophic stage and a dormant cyst stage in its life cycle. The trophozoites measure $15-60 \mu \mathrm{m}$ in diameter and are characterised by a round nucleus with a large, spherical, densely staining nucleolus. Binucleate forms are occasionally seen. In some cases the nucleus may have more than one nucleolus and this feature distinguishes B. mandrillaris amoebae from Acanthamoeba, especially in tissue sections [7,10]. During the early stages of mitosis the nucleolus and nuclear membrane both remain intact, but disappear as mitosis progresses. The nucleus is surrounded by abundant cytoplasm containing empty vacuoles, numerous mitochondria, ribosomes and endoplasmic reticulum. The trophozoites form broad pseudopodia and move slowly. Occasionally, the amoebae produce finger-like pseudopodia and exhibit spider-like walking movement across the floor of the tissue culture cells on which they are feeding [11]. The cysts of $B$. mandrillaris are usually spherical and measure $6-30 \mu \mathrm{m}$ in diameter, with a mean of $15 \mu \mathrm{m}$. They are usually uninucleate and possess a layer of refractile granules beneath the inner cyst wall. Under the optical microscope they resemble Acanthamoeba cysts with an outer wrinkled wall (the ectocyst) and an inner thin wall (the endocyst), but ultrastructurally they are tripartite with an additional thick, amorphous, fibrillar middle layer, the mesocyst [11].

N. fowleri and Acanthamoeba spp. are widely distributed in fresh water, soil and dust throughout the world, thus providing a potential source of infection for man and other animals [5-7, 12]. The environmental niche of $B$. mandrillaris is not known, but it is believed to occupy the same locations as $N$. fowleri and Acanthamoeba spp. 
Unlike Acanthamoeba spp. and N. fowleri, B. mandrillaris does not grow on non-nutrient agar plates coated with gram-negative bacteria [11]. It is probably for this reason that $B$. mandrillaris has not been isolated from nature, as free-living amoebae are usually isolated by inoculating environmental samples on bacteria-covered agar plates. B. mandrillaris grows well at $35-37^{\circ} \mathrm{C}$ on monkey kidney cells or human lung fibroblasts, as well as in a complex chemical medium containing fetal bovine serum [13], which is helpful in the screening of isolates for drug susceptibility and potential treatments $[13,14]$.

Experimental animals such as out-bred mice have been infected by intranasal instillation of culture-grown $B$. mandrillaris, resulting in disease similar to that seen in man and death of the animals $[10,11]$. A congenitally immunodeficient mouse model has also been described, confirming the 'opportunist' characteristics of $B$. mandrillaris infection [15].

\section{Clinical features and diagnosis}

Clinically, encephalitis due to $B$. mandrillaris resembles that caused by Acanthamoeba, namely the presence of a single or multiple space-occupying lesions. Neurologically, hemiparesis, aphasia and seizures may appear early. Personality and mental status abnormalities may be present, associated with headache, stiff neck and cranial nerve palsies mainly affecting the third and sixth cranial nerves. Cerebellar ataxia, diplopia and low grade fever have been reported in some cases. These symptoms closely mimic - and are often mistaken for - a bacterial or viral encephalitis, leptomeningitis and tuberculous meningitis. Raised intracranial pressure can lead to papilloedema, coma, herniation of cerebellar tonsils and death. The direct cause of death is usually acute bronchopneumonia, hepatic or renal failure, septicaemia, and brain oedema leading to uncal and cerebellar tonsillar herniation $[5,16-23]$.

The diagnosis is made when the amoebic trophozoites and cysts are identified in tissues or the agent is isolated in culture. Brain and skin biopsies are useful for culture and for histological examination with haematoxylin and eosin or special stains like Gomori's methenamine silver and periodic acid-Schiff. However, immunohistological techniques, e.g., indirect immunofluorescence assay, or electron microscopy are necessary to identify $B$. mandrillaris in tissue sections [511, 16-24]. Molecular biological methods such as the PCR or oligonucleotide probes are not available at the present time. Biopsy or autopsy tissue fixed in $10 \%$ buffered formalin or in Karnovsky's fixative can be embedded in plastic for electron microscopy to demonstrate the characteristic ultrastructural features of trophozoites and cysts [5]. A serum antibody test that may be helpful in the diagnosis of $B$. mandrillaris infection has been developed [25].

Cerebrospinal fluid (CSF) obtained by lumbar puncture may show lymphocytic pleocytosis, normal or slightly low glucose level, and mild elevation of proteins, usually without amoebic trophozoites or cysts. The CSF resembles that of aseptic meningitis in most cases.

Neuroimaging by computed tomography and magnetic resonance imaging of the head are helpful in the diagnosis $[5,23,24]$. Intravenous contrast material may reveal ring-enhancing masses suggestive of a brain abscess or a brain tumour $[5,23]$. Chest X-ray may show focal areas of consolidation in the pulmonary tissue.

\section{Pathogenesis and pathological features}

B. mandrillaris produces multifocal, subacute or chronic granulomatous encephalitis with trophozoites and cysts within the central nervous system, as well as cutaneous nodules, ulcerations and pneumonitis. Neuropathologically, infection is characterised by brain oedema and a subacute necrotising haemorrhagic encephalitis. A modest lymphocytic infiltrate is often present, depending on the immunological status of the host. This is usually composed of CD4 and CD8 T cells and B lymphocytes, together with a few plasma cells, macrophages and multinucleate giant cells. Amoebic trophozoites and cysts are usually present within perivascular spaces and within the necrotic CNS parenchyma. Focal chronic leptomeningitis may be seen in the areas near the parenchymal lesions. Arteritis with trophozoites and cysts may be seen in the same areas. Pneumonitis, and ulcerative dermatitis with the presence of trophozoites and cysts have also been described [5]. Invasion and penetration into the CNS probably arises by haematogenous spread from a primary focus of infection in the lower respiratory tract or skin [5]. The ulcerated skin lesions may serve as a portal of entry of the amoeba or may represent 'terminal' haematogenous dissemination of the infection.

B. mandrillaris can infect healthy and immunosuppressed hosts of both sexes, and there may be no history of swimming or exposure to contaminated water. Encephalitis due to $B$. mandrillaris usually runs a long protracted and insidious clinical course with an unknown incubation period, that is definitely longer than 10 days and may last up to several months.

\section{Treatment, control and prognosis}

There is presently no known effective treatment for encephalitis due to $B$. mandrillaris and the prognosis is very poor. Experimental studies indicate that the 
amoeba is sensitive to pentamidine isethionate, azithromycin and clarithromycin [13,14]. One patient with biopsy-confirmed $B$. mandrillaris encephalitis survived after treatment with the following regimen: clarithromycin $500 \mathrm{mg}$ three times a day; fluconazole $400 \mathrm{mg}$ daily; sulphadiazine $1.58 \mathrm{~g}$ every $6 \mathrm{~h}$, and 5fluorocytosine $1.5 \mathrm{~g}$ every $6 \mathrm{~h}$. However, the patient suffered severe neurological deficits (Dr T. Deetz, Santa Cruz Medical Clinic, CA, USA, personal communication to G.S.V.).

We thank Mary Bartlett and Rizwan Aslam for editorial assistance.

\section{A. JULIO MARTÍNEZ and GOVINDA S. VISVESVARA*} University of Pittsburgh Medical Center (UPMC), Pathology Department, Neuropathology Division, Presbyterian Hospital, Pittsburgh, PA 15213, USA

(e-mail: martinez@np.awing.upmc.edu), and ${ }^{*}$ Division of Parasitic Diseases, National Center for Infectious Diseases, Centers for Disease Control and Prevention, Atlanta, GA 30333, USA (e-mail: gsv@cdc.gov) Corresponding author: Dr A. J. Martínez

\section{References}

1. Culbertson CG. Pathogenic Acanthamoeba (Hartmannella). Am J Clin Pathol 1961; 35: 195-202.

2. Culbertson CG, Smith JW, Minner JR. Acanthamoeba: observations on animal pathogenicity. Science 1958; 127: 1506.

3. Fowler M, Carter RF. Acute pyogenic meningitis probably due to Acanthamoeba sp: preliminary report. BMJ 1965; 2: 740742

4. Butt CG. Primary amebic meningoencephalitis. $N$ Engl J Med 1966; 274: 1473-1476.

5. Martínez AJ, Visvesvara GS. Free-living, amphizoic, and opportunistic amebas. Brain Pathol 1997; 7: 583-598.

6. Martínez AJ, Visvesvara GS, Chandler FW. Free-living amebic infections. In: Connor DH, Chandler FC, Schwartz DA, Manz HJ, Lack EE (eds) Pathology of infectious diseases, vol II. Stamford, CT, Appleton \& Lange. 1997: 1163-1176.

7. Visvesvara GS, Stehr-Green JK. Epidemiology of free-living ameba infections. J Protozool 1990; 37: 25S-33S.

8. Rideout BA, Gardiner CH, Stalis IH, Zuba JR, Hadfield T, Visvesvara GS. Fatal infections with Balamuthia mandrillaris (a free-living amoeba) in gorillas and other Old World primates. Vet Pathol 1997; 34: 15-22.

9. Kinde H, Visvesvara GS, Barr BC, Nordhausen RW, Chiu PHW. Amebic meningoencephalitis caused by Balamuthia mandrillaris (leptomyxid ameba) in a horse. $J$ Vet Diagn Invest 1998; 10: 378-381.

10. Visvesvara GS, Martínez AJ, Schuster FL et al. Leptomyxid ameba, a new agent of amebic meningoencephalitis in humans and animals. J Clin Microbiol 1990; 28: 2750-2756.

11. Visvesvara GS, Schuster FL, Martinez AJ. Balamuthia mandrillaris, N. G, N. Sp., agent of amebic meningoencephalitis in humans and other animals. J Eukaryot Microbiol 1993; 40: $504-514$.

12. John DT. Opportunistically pathogenic free-living amebae. In: Kreier JP, Baker JR (eds) Parasitic protozoa, vol III, 2nd edn. San Diego, Academic Press. 1999: 143-246.

13. Schuster FL, Visvesvara GS. Axenic growth and drug sensitivity studies of Balamuthia mandrillaris, an agent of amebic meningoencephalitis in human and other animals. $J$ Clin Microbiol 1996; 34: 385-388.

14. Schuster FL, Visvesvara GS. Efficacy of novel antimicrobials against clinical isolates of opportunistic amebas. J Eukaryot Microbiol 1998; 45: 612-618.

15. Janitschke K, Martínez AJ, Visvesvara GS, Schuster F. Animal model of Balamuthia mandrillaris CNS infection: contrast and comparison in immunodeficient and immunocompetent mice: a murine model of "granulomatous" amebic encephalitis. $J$ Neuropathol Exp Neurol 1996; 55: 815-821.

16. Anzil AP, Rao C, Wrzolek MA, Visvesvara GS, Sher JH, Kozlowski PB. Amebic meningoencephalitis in a patient with AIDS caused by a newly recognized opportunistic pathogen, Leptomyxid ameba. Arch Pathol Lab Med 1991; 115: 21-25.

17. Taratuto AL, Monges J, Acefe JC, Meli F, Paredes A, Martínez AJ. Leptomyxid ameba encephalitis: report of the first case in Argentina. Trans R Soc Trop Med Hyg 1991; 85: 77.

18. Gordon SM, Steinberg JP, DuPuis M, Kozarsky P, Nickerson JF, Visvesvara GS. Cultural isolation of Acanthamoeba sp. and Leptomyxid amebas from patients with amebic meningoencephalitis, including two patients with AIDS. Clin Infect Dis 1992; 15: 1024-1030.

19. Griesemer DA, Barton LL, Reese CM et al. Amebic meningoencephalitis caused by Balamuthia mandrillaris. Pediatr Neurol 1994; 10: 249-254

20. Reed RP, Cooke-Yarborough CM, Jaquiery AL et al. Fatal granulomatous amoebic encephalitis caused by Balamuthia mandrillaris. Med J Aust 1997; 167: 82-84.

21. Denney CF, Iragui VJ, Uber-Zak LD et al. Amebic meningoencephalitis caused by Balamuthia mandrillaris: case report and review. Clin Infect Dis 1997; 25: 1354-1358.

22. Recavarren-Arce S, Velarde C, Gotuzzo E, Cabrera J. Amoebic angeitic lesions of the central nervous system in Balamuthia mandrillaris amoebiasis. Hum Pathol 1999; 30: 269-273.

23. Lowichik A, Rollins N, Delgado R, Visvesvara GS, Burns DK. Leptomyxid ameba meningoencephalitis mimicking brain stem glioma. Am J Neuroradiol 1995; 16 (4 Suppl): 926-929.

24. Katz JD, Ropper AH, Adelman L, Worthington M, Wade P. A case of Balamuthia mandrillaris meningoencephalitis. Arch Neurol 2000; 57: 1210-1212.

25. Huang ZH, Ferrante A, Carter RF. Serum antibodies to Balamuthia mandrillaris, a free-living amoeba recently demonstrated to cause granulomatous amebic encephalitis. J Infect Dis 1999; 179: 1305-1308. 